\title{
Radar Simulator Courses
}

\section{from Captain W. Burger and Captain A. G. Corbet}

Some lessons and observations experienced during a series of radar simulator courses in the period of one year are listed below:

I. The practice of plotting is generally favoured by the students and they learn quickly how to determine which targets are of concern and should be plotted and which targets may be rejected. It has to be pointed out fairly often that recordings should be taken down in an orderly manner and that-besides the time, bearing and range of a target-the heading and speed (or log reading) of the observing vessel should be written down with each set of observations.

It is also pointed out that in the event of a collision with a ship which has been navigating recklessly, the record of targets should be retained by the master as evidence that he has done all in his power to avert collision.

2. The relative-motion plot, when carried out, is often untidy and needs supervision and improvement by the instructor during the exercise. Too many lines are drawn on the plot and too many triangles constructed even for echoes which maintain a constant direction and rate of movement.

The relative-motion plot should be understood however because, although it is less practical than a true-motion plot, it gives masters and officers a better understanding of the meaning of the motion of echoes indicated by the 'tadpole' tails on the relative-motion display. A comment which is often overheard after an alteration of course and/or speed by own vessel is 'Look, he's altered too!' A relative-motion display often misleads even the most experienced men.

3. Students learn to appreciate that targets which are moving at slow speed need several observations before their average course and speed can be determined. Still it is not essential to know the exact course of a slow moving vessel because it is not likely to present much of a hazard. Faster moving targets yield a smooth and more definite plot.

4. Great value is attached to the use of the reflection plotter especially in conjunction with stabilized displays. On an unstabilized display it is worthwhile to mark and number the echoes on the parallel index with a chinagraph pencil before a course alteration is made. Turning the parallel index through the same number of degrees as the alteration of course will assist in re-identification of the targets.

5. Officers prefer the true-motion plot to the relative-motion plot. The former plot gives a bird's-eye view of the situation and enables the master to appreciate a dangerous situation almost at a glance in much the same way as when he observes the aspect and bearings of ships in clear weather.

In coastal waters true-motion plotting on the chart of the area is distinctly valuable provided the scale of the chart is sufficiently large (i.e. about 1 in. representing 1 mile). By plotting and studying the other vessels' courses and speeds in relation to land, shoals, anchorages, pilot stations \&c. a better appreciation of what they are doing and what they might possibly do can be obtained than by plotting on plain paper. 
6. Large course alterations and round turns for collision avoidance with several ships in the vicinity is, in general, a bad practice, especially so when unstabilized displays are used. This is because one can easily mix up targets and lose track of them as the screen becomes blurred. It is usually better to stop own ship rather than take a round turn-in any case when taking a round turn own ship probably appears stopped on the plots of the other vessels in the vicinity.

7. During simulated exercises in the English Channel with five targets simultaneously on the screen, it was found that, in the majority of cases, alterations of course again and again do not save time. Vessels which maintain their courses and take avoiding action by reducing speed and stopping as required generally make better overall progress.

8. As soon as the value of the stabilized display is appreciated, students are reluctant to go back to the head-up unstabilized display. A good combination is the relative display together with the reflection plotter and a separate true-motion plot on a much larger scale. The favourite combination is the true-motion radar display with a reflection plotter and, if required, a separate relative-motion plot of targets which appear to be converging towards one's own ship. A drawback of true-motion radar at present is that it is not as reliable-technically-as the relative-motion radar; targets appear to drift if own ship's course and speed is not portrayed correctly. However, students frequently complain of drifting of echoes of stationary targets on the true-motion display without realizing that the actual reason of this is often due to changing of the range scales. For example, switching down to a lower range scale and then back to the longer range scale will always introduce a displacement of these and all other echoes into the direction of own course on the longer scale.

9. Not enough attention is paid by the student to the proper timing of resetting the picture when using a true-motion display. Often re-setting is left too late. This is mainly because, for the majority of masters and officers the simulator course has provided their first experience of a true-motion display. It is notable how rapidly the true-motion presentation is understood and the few extra controls mastered.

I 0 . It is beneficial for students if, on occasions, the instructor changes over, without warning, the officer at the plot with the officer at the display position. This simulates a change of watch in the middle of a situation as often occurs at sea.

I I. Headreach and stopping times are estimated poorly in some cases and students watch the speed dropping with irritating impatience. Rudder movements (hard over each way to reduce speed) are recommended in such cases.

I 2. Even the use of chinagraph pencils need to be demonstrated. Marks should be 'painted on' as if using a brush. Some colours are easier to put on than others. Nothing is more frustrating than a constant breaking of points.

13. Emphasis should be laid on the sounding of fog signals (buzzers). The instructor should announce the direction from which they are coming. The latter procedure is extremely important for close quarter manœuvres. Some targets may be stopped, others may have way upon them.

14. Collisions or near-collisions have occurred owing to:

(a) Too much speed.

(b) Forgetting about the existence of a shadow sector ahead.

(c) Wrong interpretation of tadpole tails on relative-motion displays. 
Tadpole tails of ships on slightly divergent courses but going in approximately the same direction seem to be confusing and wrong manœurres have been executed as a result thereof.

1 5. The post-mortem is of immense value, in particular, when the movement of the vessels are repeated step-by-step on the master (true motion) plot-kept by the instructor-by means of small wooden models painted in different colours. This gives a chance to the men in command of vessels to discuss their actions and to learn the opinion of others, an occasion which would never occur on board. The movement of other 'vessels' (clear from the fog bank or without radar) will also become clear after the overall situations have been revealed. In some exercises a fast moving target sounding no fog signals and representing a ship commanded by an irresponsible (or unenlightened) master is presented on the display. This can create an interesting and realistic situation and helps to stimulate discussion afterwards.

16. Except from the pictorial point of view simulated coast lines are of little value. It would seem to be better to have more targets at one's disposal (ten, for example). Some of these targets could be used to represent landpoints, lightvessels \&c. and exercises could then be carried out for any part of the world.

In conclusion one may say that the development of the radar simulator has proved to be a tremendous advance in radar training.

It is felt that junior officers can also benefit from the simulator courses because although they may not be directly concerned with the actual manceuvring of ships for some years, they are often required to plot under pressure and the simulator course can give them practice at this. The simulator also enables the student to acquire a fuller understanding of the different types of display presentation he might encounter on various ships.

\section{TRAFFIC REGULATION AT SEA}

The Maritime Safety Committee of the Inter-Governmental Maritime Consultative Organization considered the Report of the Dover Strait Working Group set up by the British, French and German Institutes of Navigation, at its session which ended on 25 January. Members of the Safety Committee were, in general, in agreement with the recommendations of the Group that a system of routing should be established and that the navigational marks and other aids in the Strait should be improved. The Committee considered, however, that the report required further study by governments before definite views could be formed; account would also have to be taken of the result of the enquiry now being addressed to masters of ships by the International Chamber of Shipping through its members.

In the meantime, the Committee asked the IMCO Secretariat to keep in close touch with the Institutes of Navigation and to collect all the information on further developments which might become available. 